\title{
Fast Elastic Registration for Adaptive Radiotherapy
}

\author{
Urban Malsch $^{1}$, Christian Thieke ${ }^{2,3}$, and Rolf Bendl ${ }^{1}$ \\ ${ }^{1}$ Department of Medical Physics \\ u.malsch@dkfz.de \\ ${ }^{2}$ Clinical Cooperation Unit Radiooncology, DKFZ Heidelberg \\ ${ }^{3}$ Department of Radiation Oncology, University of Heidelberg, Germany
}

\begin{abstract}
A new method for elastic mono-modal image registration for adaptive fractionated radiotherapy is presented. Elastic registration is a prerequisite for many medical applications in diagnosis, therapy planning, and therapy. Especially for adaptive radiotherapy efficient and accurate registration is required. Therefore, we developed a fast block matching algorithm for robust image registration. Anatomical landmarks are automatically selected at tissue borders and relocated in the frequency domain. A smooth interpolation is calculated by modified thinplate splines with local impact. The concept of the algorithm allows different handling of different image structures. Thus, more features were included, like handling of discontinuities (e.g. air cavities in the intestinal track or rectum, observable in only one image), which can not be registered in a conventional way. The planning CT as well as delineated structures of target volume and organs at risks are transformed according to deviations observed in daily acquired verification CTs prior each dose fraction. This way, the time consuming repeated delineation, a prerequisite for adaptive radiotherapy, is avoided. The total calculation time is below 5 minutes and the accurateness is higher than voxel precision, which allows to use this tool in the clinical workflow. We present results of prostate, head-and-neck, and paraspinal tumors with verification by manually selected landmarks. We think this registration technique is not only suitable for adaptive radiotherapy, but also for other applications which require fast registration and possibilities to process special structures (e.g. discontinuities) in a different way.
\end{abstract}

\section{Introduction}

Recent developments in radiotherapy allow a high precision in shape of dose distributions in three dimensional target volumes (e. g. tumor), while high gradients reduce the applicated dose in other regions (e. g. organs at risk: OAR). Currently correct positioning of the patient is assured by fixation techniques (head masks, vacuum pillow, ...). With recently available in-room 3D imaging techniques, e. g. ConeBeam CT (kilovoltage-Source attached at Linac-gantry) or CT scanner on rails (e.g. Siemens Primatom) global setup errors can be determined by rigid registration between planning and verification $\mathrm{CT}$ images, which are acquired 
directly before therapy 1]. Rigid registration can not compensate complexer deviations, e.g. organ movements, different fillings of hollow organs (bladder, rectum, lung, ...), tissue dependent reaction to the irradiation, or other motion artifacts, e.g. caused by respiration during therapy. So far, security margins around the tumor and OARs are used to consider these uncertainties. At the same time, however, healthy tissue next to the tumor is also irradiated with a therapeutical dose level and possibly damaged. A novel strategy of dynamic adaptation of an initial plan to daily changes in size, shape, and position of the target volume and critical structures allows a reduction of security margins and thus a reduction of normal tissue complication probability while preserving or increasing tumor control probability 2]. This adaptation has to be done within less than 15 minutes, in order to keep up with physiological changes (e. g. different filling of the bladder) and clinical workflow. So far no automatic segmentation tool can produce a complete delineation within this time. Therefore, we propose a different strategy by elastic registration of spatial deviations between planning and verification CT image. We apply the measured distortions to the already segmented patient model to produce adapted delineations.

Several registration techniques were developed in the past decades [3], like optical flow [4, demon algorithm [5], viscose fluid 667, free form deformations [8], or block matching [9]. Most of them are rather expensive in terms of computation time. For adaptation of therapy plans, especially calculation time is an important constraint. We use a block matching algorithm similar to that one proposed by Rösch et al [9] with some modifications, which make the algorithm fast and allow the integration of additional features, like handling of discontinuities in the deformation field, e.g. due to moving air cavities in rectum.

\section{Method}

Our elastic registration algorithm is divided into three main steps:

1. Automatic identification of a few landmarks at positions $\boldsymbol{p}_{i}=\left(p_{i, x}, p_{i, y}, p_{i, z}\right)$, $(i=1 \ldots N)$ in first image.

2. Calculation of appropriate translation vectors $\boldsymbol{t}_{i}$ to corresponding positions in the other image by optimizing a similarity measure in a surrounding subregion $\Omega_{\boldsymbol{p}_{i}}$, called templates of $\boldsymbol{p}_{i}$.

3. Interpolation for any position $\boldsymbol{q}$, which can be polygon vertices of delineated structures or all other image positions, to transform them.

These general registration steps will now be discussed in detail:

\subsection{First Step, Selection of Promising Positions}

Promising positions $\boldsymbol{p}_{i}$ are spots in an image $A$, which can presumably be recognized in the corresponding image $B$. Positions at tissue borders turned out to be especially suitable. In CT images, tissue classes (e.g. fat, muscle, bones) can be identified quickly by fixed thresholds. Border positions of these classes are 

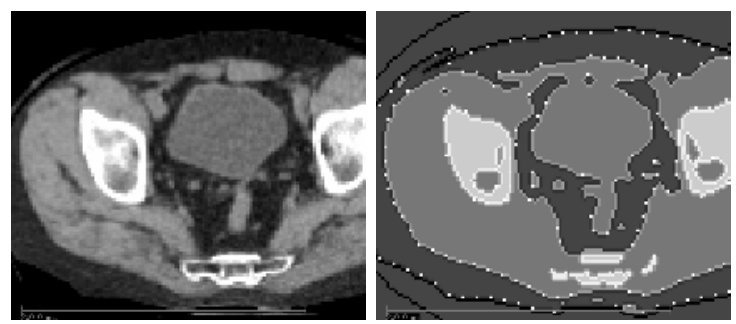

Fig. 1. (left): transversal slice of a prostate case. (right): raw tissue separation by fixed thresholds with selected template candidates.

identified by voxels with less than 6 neighbors of same class. Promising template positions $\boldsymbol{p}_{i}$ are evenly distributed at the borders with consideration of minimal distance between each other to avoid agglutinations (see Fig. 1).

\subsection{Second Step, Relocation}

Each promising position $\boldsymbol{p}_{i}$ of image $A$, representing a characteristic structure, must be relocated into image $B$ to identify the deviation $\boldsymbol{t}_{i}$ between the images at these positions.

By defining template areas $\Omega_{i}$ around each position $\boldsymbol{p}_{i}$, these sub-images can be re-identified in the corresponding image by local rigid registration $R_{i}$, to get a corresponding position $\boldsymbol{q}_{i}=\boldsymbol{p}_{i} R_{i}$. Several similarity measurements were proposed in literature to identify best correspondences [10. We use the maximization of cross correlation coefficients $\Theta_{C}$, calculated in the frequency domain, which computes transformation parameter within a bounded local region. A subsequent normalized $\Theta_{C}$ is used as an absolute measure of conformity, which allows the identification of doubtful correspondences. Cross correlation $\Theta_{C}$ between a small domain $\Omega_{A(\boldsymbol{p})}$ at position $\boldsymbol{p}$ of image $A$ and a rigidly displaced domain $\Omega_{B(p R)}$ of image $B$ is defined by

$$
\Theta_{C}\left(\Omega_{A(\boldsymbol{p})}, \Omega_{B(\boldsymbol{p} R)}\right)=\sum_{\boldsymbol{x} \in \Omega_{(A, B)}}\left(\Omega_{A(\boldsymbol{p})}(\boldsymbol{x})-\bar{\Omega}_{A(\boldsymbol{p})}\right)\left(\Omega_{B(\boldsymbol{p} R)}(\boldsymbol{x})-\bar{\Omega}_{B(\boldsymbol{p} R)}\right)
$$

and the normalized correlation $\Theta_{C}$ is defined by

$$
\Theta_{N C}\left(\Omega_{A(\boldsymbol{p})}, \Omega_{B(\boldsymbol{p} R)}\right)=\frac{\Theta_{C}\left(\Omega_{A(\boldsymbol{p})}, \Omega_{B(\boldsymbol{p} R)}\right)}{\sqrt{\Theta_{C}\left(\Omega_{A(\boldsymbol{p})}, \Omega_{A(\boldsymbol{p})}\right) \Theta_{C}\left(\Omega_{B(\boldsymbol{p} R)}, \Omega_{B(\boldsymbol{p} R)}\right)}}
$$

Eq.(11) can be calculated efficiently in the frequency domain by fast Fourier transformation (FFT) of the areas $\Omega_{A(\boldsymbol{p})}$ and $\Omega_{B(\boldsymbol{p} R)}$ :

$$
\Theta_{C}\left(\Omega_{A}, \Omega_{B}\right)=\operatorname{FFT}^{-1}\left(\operatorname{FFT}\left(\Omega_{A}\right)\left(\operatorname{FFT}\left(\Omega_{B}\right)^{*}\right)\right)
$$

with $X^{*}$ conjugate complex of $X$. The displacement of a feature is identified at the maximum of $\Theta_{C}$ (see Fig. 22). In our application it is sufficient to consider translations only, since a rotation of larger structures can be described by different displacements of their surface points. 


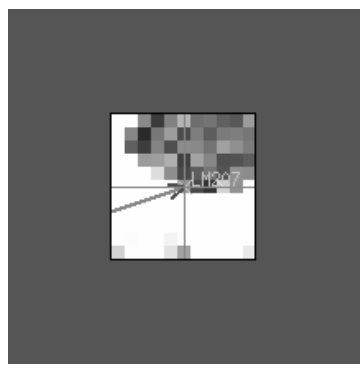

(a)

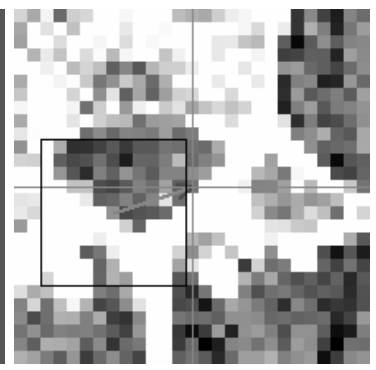

(b)

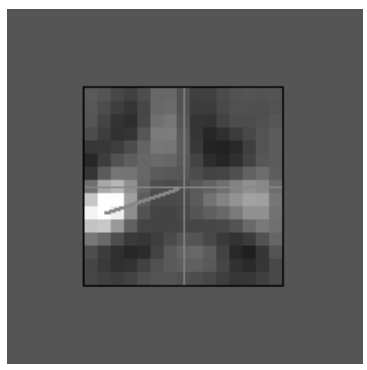

(c)

Fig. 2. (a) a feature in image $A$ at position $\boldsymbol{p}_{i}$ (here located at border of a vertebral body), (b) searching area at same position in corresponding image $B$ and (c) surrounding correlation values (calculated in frequency domain). The bright spot indicates best translation. Size of feature: $11 \times 11 \times 7$ voxel (padded with zero values), size of search region: $27 \times 27 \times 17$. Maximal detectable displacements are $t_{x, y, z}= \pm(8,8,5)$.

\subsection{Third Step, Interpolation}

In this step, elastic transformation of delineated planning structures (VOIs) are calculated. We define VOIs by closed polygons, located in transversal CT slices. Thus, vectors for all polygon vertices must be calculated to transform them to the verification image. If the whole planning CT should be transformed, too (e. g. to verify the registration), translation vectors for all voxels must be interpolated as well. Regularly arranged translation vectors can be interpolated quickly, e. g. by B-splines or trilinear interpolation. But since templates are arbitrarily arranged, we use modified thin-plate splines (TPS) 11 to interpolate a translation vector field $T(A, B)$. Each translation $\boldsymbol{t}=\left(t_{x}, t_{y}, t_{z}\right) \in \mathbb{R}^{3}$, located at any position $\boldsymbol{q}=\left(q_{x}, q_{y}, q_{z}\right) \in \mathbb{R}^{3}$, is defined by:

$$
\boldsymbol{t}(\boldsymbol{q})=a_{0}+a_{x} q_{x}+a_{y} q_{y}+a_{z} q_{z}+\sum_{i=1}^{n} w_{i, x y z} \cdot u\left(\boldsymbol{q}-\boldsymbol{p}_{i}\right)
$$

with the radial base function $u(\boldsymbol{t})=|\boldsymbol{t}|=\sqrt{t_{x}^{2}+t_{y}^{2}+t_{z}^{2}}$, which corresponds to the minimization of the bending energy of a thin metal plate in $3 \mathrm{D}$ case. The TPS interpolation is global in nature and one can not avoid the global effect of each template. Among others, this means that the transformation at a position $\boldsymbol{q}$ is affected by each anchor point $\boldsymbol{p}_{i}$. This is clear, because the number of coefficients, calculated by matrix inversion and multiplications with anchor positions, are equal to the number of anchors. Since calculation time for inverting the matrices to calculate the affine and elastic coefficients $\left(a_{0, x, y, z}\right.$ and $\left.w_{i}\right)$ increase quadratically with the number of templates, we use a series of locally defined TPS to speed up the calculation and to reduce the influence of far-off image positions. As a result, one translation vector does not affect all image positions, but only its nearby image fraction. These image fractions are determined in a recursive process: we start with the whole image as the first current fraction. 


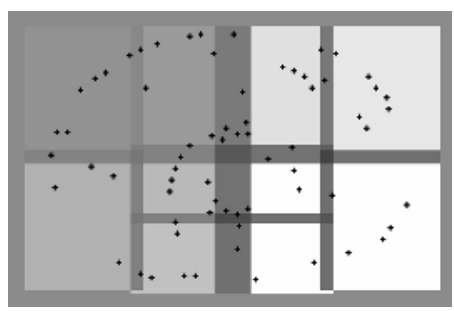

Fig. 3. Block TPS. The initial image is divided in a recursive process, as long as a fraction contains more than $10 \%$ of the translation vectors (black dots). An overlap of $10 \%$ of the fractions guarantees a smooth transition.

If the current fraction contains more than a predefined number of template positions $\boldsymbol{p}_{i}$, this fraction is divided into two halves (first along $x$-, second along $y$ - and last along $z$-direction) and that strategy is recursively applied to both fractions.

Each fraction is handled by an individual TPS interpolation. To guarantee a smooth transition between all fractions, an overlap of $10 \%$ is defined (Fig. 3). Inside the overlapping regions vectors are calculated separately by all related fractions. The linear interpolation of all involved fractions is used as final value. The number of calculated translation vectors is slightly increased due to overlapping regions. But overall calculation time is reduced markedly, since the number of TPS coefficients for each calculation is reduced.

\subsection{Discontinuities}

Discontinuities are a result of features, observable in only one image, e.g. gas filled cavities inside the rectum or colon. No adequate maximization of $\Theta_{C}$ can be found in these regions. However, especially for usage in adaptive radiotherapy for prostate cancer, air cavities deform strongly proximate image structures like prostate and bladder. The method presented so far has its limitations under these conditions, but a separate processing at these regions allows to overcome this drawback:

After Gaussian filtering, air cavities are easily detected by an appropriate threshold. Corresponding pairs of landmarks at border and center of cavities allow a constriction by TPS interpolation. Border positions $\boldsymbol{p}_{i}$ are detected in the same manner as described in Chap. 2.1. To determine in 3D corresponding positions $\boldsymbol{q}_{i}$ at the exact center, a 3D distance map $D$ is calculated, which indicates for each voxel inside of the cavity the distance to the rectum wall. Therefore, the maximal value of the distance map along the path perpendicular to the wall indicates corresponding center positions:

$$
\boldsymbol{q}_{i}=\boldsymbol{p}_{i}+r_{0} \nabla A\left(\boldsymbol{p}_{i}\right), \text { with } r_{0}=\underset{r}{\operatorname{argmax}} D\left(\boldsymbol{p}_{i}+r \nabla A\left(\boldsymbol{p}_{i}\right)\right)
$$

$\nabla A\left(\boldsymbol{p}_{i}\right)$ is the gradient at border position $\boldsymbol{p}_{i}$ in the image $A$. To assure that the constriction will have only local influence, the TPS-interpolated vectors are 


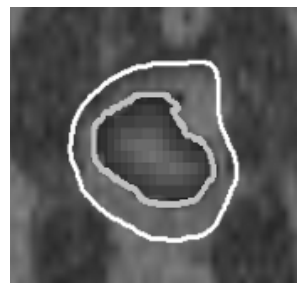

(a)

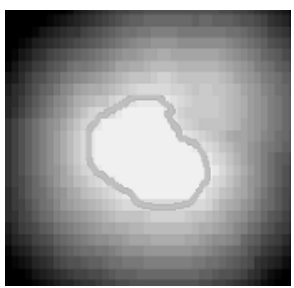

(b)

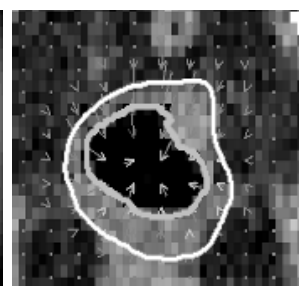

(c)

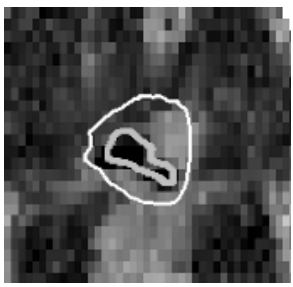

(d)

Fig. 4. All images show the same frontal slice of an air cavity in the rectum. White contour: original delineated rectum, gray contour: automatically segmented air cavity. (a) distance map to indicate distances from border to center of the air cavity, (b) distances outside of air cavity. (c) TPS interpolation, locally limited to a near neighborhood by weighting resulting vectors with distance map (b). (d) constricted air cavity and transformed delineations.

multiplied by a weighting factor, which is reciprocal to its distance to the air cavity. In addition, to avoid misleading distortions of bony structures in the direct neighborhood of air cavities, additional fixation points are inserted to forbid the deformation of these structures.

\section{Results}

\subsection{Quantitative Measurements}

Quantitative verification of elastic registration results is as complex as its calculation. Since every image position is calculated individually, for every point of the image an individual error is present. If it would be possible to measure the deviation between calculated vectors and real movements exactly one would certainly consider this deviation. For patient data no objective gold standard exists, even if fiducials like gold seeds would have been implanted they would show the real motion only at a limited number of positions. However, usually it is possible to identify a series of anatomical landmarks in both data sets quite well to describe deviations by distances between corresponding positions. We manually defined those landmarks in a series of 5 test cases. These cases

Table 1. Maximal absolute deviations $(t x, t y, t z)$ between both original images and maximal absolute error in automatic calculated deviations $(d x, d y, d z)$ (in $\mathrm{mm})$

\begin{tabular}{ll|ccc|ccc|c}
\hline \# & Tumor & \multicolumn{3}{|c|}{$\mathrm{LM}_{m}$} & \multicolumn{3}{|c|}{$\mathrm{LM}_{a}-\mathrm{LM}_{m}$} & $n_{i}$ \\
\hline & & $\max _{|t x|}$ & $\max _{|t y|}$ & $\max _{|t z|}$ & $\max _{|d x|}$ & $\max _{|d y|}$ & $\max _{|d z|} \mid$ & \\
1 & Prostate & 4.4 & 5.4 & 8.5 & 1.9 & 2.2 & 3.1 & 67 \\
2 & Parasp. & 3.4 & 5.4 & 9.0 & 2.0 & 2.0 & 2.9 & 59 \\
3 & Parasp. & 14.2 & 5.4 & 8.3 & 3.2 & 2.0 & 2.9 & 63 \\
4 & H \& N & 6.1 & 9.3 & 6.4 & 2.1 & 2.1 & 3.2 & 78 \\
5 & H \& N & 4.4 & 19.2 & 6.3 & 2.0 & 2.9 & 3.0 & 79 \\
\hline
\end{tabular}



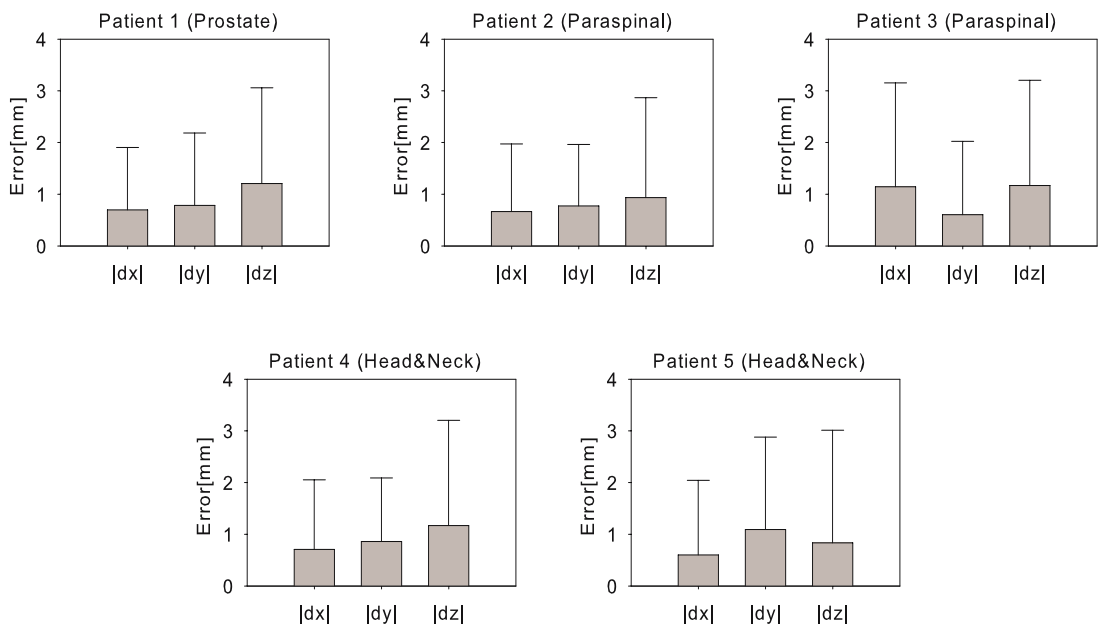

Fig. 5. These bar charts show the mean errors in $x, y$ and $z$-direction for five selected patients and maximal errors (whisker caps). The errors show differences between manually selected landmarks and those vectors of the automatically calculated translation vector field at same positions.

were carefully selected to assure that sufficient large displacements are present. Manually selected landmarks allow a semi-quantitative quality control of our strategy. This procedure was applied to one prostate, two paraspinal and two head-and-neck cases to show robustness of the algorithm. The results of five sets with $n_{1} \ldots n_{5}$ pairs of landmarks are represented in Table1, First column $\left(\mathrm{LM}_{m}\right)$ describes deviations between both original images by maximal distances. We observed deviations of about $1 \mathrm{~cm}$, in maximum $14.2 \mathrm{~mm}$ and $19.2 \mathrm{~mm}$ (patient 3 and 5). Maximal absolute differences $\left(d_{x}, d_{y}, d_{z}\right)$ between automatically calculated deviations $\mathrm{LM}_{a}$ and manually selected landmarks $\mathrm{LM}_{m}$ are represented in second column, which describe maximal error of each registration result. Highest errors in $x$ - and $y$-direction $(3.2 \mathrm{~mm}$ and $2.9 \mathrm{~mm})$ were observed in registration of patient 3 and 5, having shown the strongest deviations at all. Deviations in $z$-direction were about $3.0 \mathrm{~mm}$. Results of automatic registration is acceptable, since mean error is below voxel size $\left(2 \times 2 \times 3 \mathrm{~mm}^{3}\right)$. However, some outliers were observed (up to $3.2 \mathrm{~mm}$ ), visualized by its maximum values with whisker caps in the bar charts of Figure 5. Most of these outliers are a result of landmarks, located at positions with low clinical importance, e. g. at patient's surface. The presented approach calculates an elastic transformation between different CT datasets. The algorithm was designed to reuse pre-segmented structures by adapting them to each therapy situation. Overall, the main objective was a low calculation time, since the algorithm should be used in a small time frame (below 10 mins). This approach allows to handle image structures, which can not be processed by conventional registration, in a different manner. We included a procedure to constrict air cavities, which represent discontinuities between two images and can not be handled in the conventional way. With the current 
implementation, a registration of a normal CT $(256 \times 256 \times 50)$ is done in less than 5 minutes with accurateness below voxel precision in clinical relevant structures (measured by comparison with manually selected landmarks). This allows us to use our tool for selected cases in clinical routine. Even breathing motion can be detected in 4D CT-images to individualize initial security margins. However, the most time consuming part is still the search for correspondences (half of the calculation time). In the next step, we want to implement this part in parallelized algorithms, which should speed up the total calculation time.

\section{References}

1. Thieke, C., Malsch, U., Bendl, R., Thilmann, C.: Kilovoltage CT using a linac-CT scanner combination (accepted). Br. J. Radiol. (2006)

2. Yan, D., Vicini, F., Wong, J., Martinez, A.: Adaptive radiation therapy. Phys. Med. Biol. 42 (1997) 123-132

3. Zitová, B., Flusser, J.: Image registration methods: a survey. Image and Vision Computing 21 (2003) 977-1000

4. Schunck, B.G., Horn, B.K.P.: Determining optical flow. Artificial Intelligence $\mathbf{1 7}$ (1981) 185-204

5. Thirion, J.P.: Image matching as a diffusion process: an analogy with maxwell's demons. Med. Image Anal. 2 (1998) 243-260

6. Christensen, G.E., Joshi, S.C., Miller, M.I.: Volumetric transformation of brain anatomy. IEEE Trans. Med. Imaging 16 (1997) 864-877

7. Foskey, M., Davis, B., Goyal, L., Chang, S., Chaney, E., Strehl, N., Tomei, S., Rosenman, J., Joshi, S.: Large deformation three-dimensional image registration in image-guided radiation therapy. Phys. Med. Biol. 50 (2005) 5869-5892

8. Rueckert, D., Sonoda, L.I., Hayes, C., Hill, D.L., Leach, M.O., Hawkes, D.J.: Nonrigid registration using free-form deformations: application to breast MR images. IEEE Trans. Med. Imaging 18 (1999) 712-721

9. Rösch, P., Mohs, T., Netsch, T., Quist, M., Penney, G.P., Hawkes, D.J., Weese, J.: Template selection and rejection for robust non-rigid $3 \mathrm{D}$ registration in the presence of large deformations. Proceedings of the SPIE (2001) 545-556

10. Hill, D.L., Batchelor, P.G., Holden, M., Hawkes, D.J.: Medical image registration. Phys. Med. Biol. 46 (2001) R1-45

11. Bookstein, F.: Principal warps: Thin-plate splines and the decomposition of deformations. IEEE Trans. Pattern Anal. Mach. Intell. 11 (1989) 567-585 